\title{
The Waveform Digitiser of the Double Chooz Experiment: Performance and Quantisation Effects on PhotoMultiplier Tube Signals
}

\author{
Y. Abe $^{d}$, T. Akiric ${ }^{c}$ A. Cabrera ${ }^{a}$, B. Courty ${ }^{a}$, J. V. Dawson ${ }^{a *}$, L. F. G. Gonzalez ${ }^{a, b}$, \\ A. Hourlier ${ }^{a}$, M. Ishitsuka ${ }^{d}$, H. de Kerret ${ }^{a}$, D. Kryn ${ }^{a}$, P. Novella $^{a}$, M. Obolensky ${ }^{a}$, \\ S. Perasso ${ }^{a}$, A. Remoto ${ }^{a, e}$, R. Roncin ${ }^{a}$ \\ ${ }^{a}$ Laboratoire Astroparticule et Cosmologie, 10 rue Alice Domon et Léonie Duquet, 75205 Paris, \\ France \\ ${ }^{b}$ Universidade Estadual de Campinas-UNICAMP, Campinas, SP, Brazil \\ ${ }^{c}$ Duke University, Department of Physics, Durham, North Carolina, U.S.A \\ ${ }^{d}$ Department of Physics, Tokyo Institute of Technology, Tokyo, 152-8551, Japan \\ ${ }^{e}$ Laboratoire d'Annecy-le-Vieux de physique des particules, 9 Chemin de Bellevue, 74941 \\ Annecy-le-Vieux, France \\ E-mail: jaime.dawson@gmail.com
}

\begin{abstract}
:
We present the waveform digitiser used in the Double Chooz experiment. We describe the hardware and the custom-built firmware specifically developed for the experiment. The performance of the device is tested with regards to digitising low light level signals from photomultiplier tubes and measuring pulse charge. This highlights the role of quantisation effects and leads to some general recommendations on the design and use of waveform digitisers.
\end{abstract}

KEYWORDS: Waveform digitisers, Flash ADC, Quantisation, Digitisation, PhotoMultiplier Tubes .

\footnotetext{
${ }^{*}$ Corresponding author.
} 


\section{Contents}

1. Introduction 1

2. Double Chooz Electronics 2

3. The Double Chooz waveform digitiser

3.1 Synchronisation

3.2 Trigger and Event Metadata

3.3 Real-Time Signal Counting 5

4. FADC Testing 5

4.1 FADC Linearity

4.2 High Frequency Correlated Noise 6

5. Digitisation 7

5.1 Quantisation 8

5.2 Experimental Study of Quantisation Effects 9

6. Simulation 12

7. Conclusion 12

\section{Introduction}

The growing usage of Waveform Digitisers in Experimental Physics applications has been made possible by the progress of Flash ADC chip industry. It is driven by two motivations: first, instead of recording separately some of the properties of the signal like timing, amplitude and charge, one can now use one single digitiser per channel to record the whole signal itself and derive its properties by software or in firmware; second, the event represented by the signal can easily be stored temporarily before the decision is taken to keep or reject it. In addition, recording signal profiles allows to exploit Pulse Shape Discrimination techniques leading to a better understanding of background events, and gives the ability to discriminate better between physics and spurious signals.

The Double Chooz experiment measures the third neutrino mixing angle $\theta_{13}$ using anti-neutrinos emitted from a nuclear power plant [1], 2]. The detector uses liquid scintillators [3] observed by 390 ten-inch low background PhotoMultiplier Tubes (PMT) (Hamamatsu R7081 [4, 5, 6]) for the neutrino target, and 78 eight-inch PMTs (Hamamatsu R1408 [7]) for the muon veto. The PMTs are operated in high gain mode since the number of photons impacting on each individual PMT is low. For an event, the charge contained in each PMT waveform is measured and the total charge 


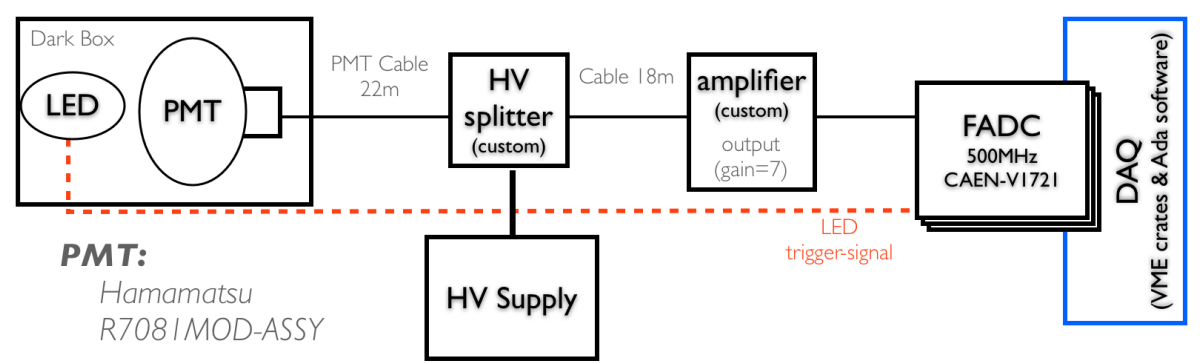

Figure 1. Electronics for the single channel test.

observed by all PMTs is used to determine the energy deposited. The energy range of the neutrino signal begins below $0.7 \mathrm{MeV}$ for the positron interaction and extends up to $10 \mathrm{MeV}$ to well contain the gamma rays resulting from neutron capture by Gadolinium. In practice, data is recorded from $300 \mathrm{keV}$ on the low energy side. On the high energy side, the detector response is linear to approximate $50 \mathrm{MeV}$ which allows sampling of important backgrounds. In this energy range, each PMT signal contains from zero to $\sim 50$ photoelectrons. Cosmic ray muons crossing the detector deposit even higher energies, saturating the waveform digitiser channels described in this article resulting in an overall non-linear energy response which extends up to $\sim 600 \mathrm{MeV}$. The recording of the PMT waveforms is therefore an important factor in the overall energy response of the detector.

In this article, we give a general description of the electronics chain involved in the digitisation of the PMT signals (Section 2); then discuss the Flash ADC hardware and describe the main features of the firmware written specifically for the experiment (Section B). Before being installed at the experimental site, a series of tests were made on each waveform digitiser card to ensure their good working condition [8]. The results of one such test, testing the linearity of all channels, are reported in Section 4.1. The response to PMT signals was explored with tests of a single channel mock-up of the full Double Chooz electronics chain. Signals cannot be perfectly recorded; there are undesirable effects such as high frequency noise (Section 4.2), ADC non-linearity, and digitisation effects which can produce subtle biases on the measurement of pulse charge. In Section 5 , we discuss the sources of bias on the measurement of pulse charge. We show that these effects can be well reproduced by simulation, and also measured in-situ with a suitable light calibration system. We also discuss the working conditions necessary to minimise their effects. Finally Section 6 shows results from a detailed simulation of a waveform digitiser.

\section{Double Chooz Electronics}

Figure 1 is a diagram of the single channel electronics. The PMTs are operated at a gain of $10^{7}$, the fast signal is decoupled from the PMT HV cable by a custom-built HV splitter. The signal from the PMT is amplified by the Double Chooz amplifier with a gain of $\sim 7$ such that after amplification the mean single photoelectron amplitude is $\sim 35 \mathrm{mV}$. The analog RMS noise level was measured as $\sim 1.2 \mathrm{mV}$ at the output of the amplifier. All PMT signals are sampled synchronously every 2 ns by the waveform digitisers. The Data Acquisition system runs via VME bus, with each crate 
controlled by a single-board computer (MVME3100 PowerPC [9]) running Debian Linux with Data Acquisition software written in Ada 2005.

The first reason to use Waveform Digitisers for the Double Chooz experiment was the desire to keep a full control on the determination of the signal properties. This means to be able to control, from the recorded data, the pulse position within the integration window, and check that the time measurement is not done on a spurious signal. Waveform digitisers also allow to obtain simultaneous digitisation and read out without dead time.

\section{The Double Chooz waveform digitiser}

Table 1. Main characteristics of the Double Chooz waveform digitiser

\begin{tabular}{|l|c|}
\hline Time resolution & $2 \mathrm{~ns}$ \\
\hline Time precision & $<5 \mathrm{ps}$ \\
\hline Amplitude resolution (LSB) & $78 \mu \mathrm{A} / 3.9 \mathrm{mV}$ \\
\hline Dynamic range & 8-bit / 20mA / 1V \\
\hline Differential Non-Linearity & $\begin{array}{c}\text { Typical maximum of }|0.16| \mathrm{LSB} \\
\text { with limits } \pm 0.6 \mathrm{LSB}\end{array}$ \\
\hline Integral Non-Linearity & $\begin{array}{c}\text { Typical maximum }|0.3| \mathrm{LSB} \\
\text { with limits } \pm 0.9 \mathrm{LSB}\end{array}$ \\
\hline SRAM Memory Buffer Size & 2 MiByte/channel \\
\hline
\end{tabular}

The waveform digitiser for the Double Chooz experiment was developed in partnership with CAEN, and is available as model Vx1721 (VME64x) or V1721[10] (legacy VME). It is based on an 8-Channel 8-bit, $500 \mathrm{MS} / \mathrm{s}$ Flash ADC (FADC). For interest, please note that a 10-bit $1 \mathrm{Gs} / \mathrm{s}$ version is now available [11].

The model used by Double Chooz (see Table 1 for main characteristics) has single ended signal inputs each with a dynamic range of approximately 1 Volt. A DC-offset can be applied individually to each channel through the use of an onboard 16-bit DAC. For the negatively-going PMT signals of Double Chooz this offset is set such that ground is found around ADC code 210, in this way allowing some room for the digitisation of positive over-shooting signals whilst maintaining a good dynamic range for the negatively-going PMT signals.

Double Chooz has developed a firmware for this board, which is different to the commercial version developed by CAEN, and is tailored for the experiment. The description below of the board functionalities is essentially the description of this firmware. Each channel has a SRAM memory buffer (of total size $2 \mathrm{MiByte}^{1}$ ) which is divided into 1024 circular memory buffers or pages. A sophisticated control logic divides memory access periodically in two different time slots, one for write and one for read, only delaying the pending read or write accesses.

The data stream is continuously written into one page until the arrival of a trigger signal. When the trigger occurs, the page is frozen and the acquisition continues without dead time by writing into the following empty page. The pages are unfrozen by the means of VME commands. All

\footnotetext{
${ }^{1} M i \equiv \times 2^{20} \sim 1.05 \times 10^{6}$
} 
pages are permanently accessible for reading through the VMEbus. The memory can be seen as a transparent FIFO: events enter the FIFO instantly on trigger and are removed instantly by a VME command. In the mean time, they can be inspected and read randomly (transparency of the FIFO). If the read-out program was not able to unfreeze the pages fast enough, so that the FIFO becomes full, dead-time would occur. This condition is detectable by software which would then raise a warning or an exception.

The front-panel clock input is used for the synchronisation of multiple cards with the Data Acquisition clock. Multiple cards can therefore be triggered simultaneously by NIM signals. Also on the front-panel are 16 programmable LVDS Input/Outputs.

The VME capabilities of the board which are implemented in the firmware include the fastest VME64x transfer protocol, 2eSST, at a maximum speed of $320 \mathrm{MiByte} / \mathrm{s}$. Geographical Addressing, available in VME64x, greatly facilitates the management of a large number of boards, allowing to automate board detection and address assignments.

\subsection{Synchronisation}

The synchronisation happens in two steps:

1. Card synchronisation: The Trigger System distributes a $62.5 \mathrm{MHz}$ (16 ns period) clock and a synchronous trigger signal. The clock is distributed as LVDS and the trigger as NIM.

2. Channel synchronisation: An onboard $\mathrm{PLL}^{2}$ produces a $500 \mathrm{MHz}$ (2 ns period) clock synchronous with the external 62.5 MHz. The trigger signal is distributed to each of four memory-management logical units (one per two channels), which all switch pages at the same time, on edges of the $62.5 \mathrm{MHz}$ clock.

The $62.5 \mathrm{MHz}$ clock gives the actual pace of the board logic. Every $16 \mathrm{~ns}$, eight FADC samples (eight measurements of the input current), done at 2 ns intervals, are stored in the SRAM memory. The maximum waveform length available is $4 \mu \mathrm{s}$ ( $2 \mathrm{kiB}$ per channel). The trigger point of each board is completely flexible, similar to the horizontal time-offset on an oscilloscope, a configurable delay can be added to move the trigger point from the end of the waveform to the start, in steps of 16 ns.

\subsection{Trigger and Event Metadata}

The Double Chooz Trigger generates information about the triggering conditions and calculates the event number. Synchronous to the trigger, this trigger data is passed to the Waveform Digitiser boards through the 16-bit LVDS input connector present on the front-panel. This information, together with the number of clock ticks since the previous trigger and an internal trigger counter, are stored by the Waveform Digitisers and one set is kept for each page. This constitutes the event metadata; it can be read from the VME, and used to categorise the event types and differentiate data handling.

\footnotetext{
${ }^{2}$ Phase-Locked Loop
} 


\subsection{Real-Time Signal Counting}

As the FADC is continuously digitising it can also be setup to monitor the signal rate on each channel. This is achieved by setting (by software) a threshold on each channel such that when it is crossed by a signal, a positive square pulse of firmware-determined width and amplitude is generated. All such signals from each channel are combined and the result is sent to the LEMO output on the front panel through a 16-bit DAC, with an amplitude which is set in the firmware by selecting which 4 bits out of the 16 are used. In this way the number of channels simultaneously firing is coded into the amplitude of an analog signal. This information per card could be used to form the system-wide Trigger condition. This is the basis of a future enhancement to the Double Chooz Trigger system.

\section{FADC Testing}

The main objective of the Double Chooz waveform digitiser is to record scintillator pulses and reconstruct the contained charge. In this section we describe the behaviour of the waveform digitiser, with particular emphasis on the sources of bias on the charge measurement.

FADCs convert analog waveforms to digital form, by using a linear voltage ladder with comparators at each rung to compare input voltages to successive reference voltages. The output of the comparators are fed into a digital encoder which outputs binary values. The terms 'ADC code' or 'ADC count' will be used hereafter to denote these output values 0 to 255 for the 8-bit FADC. The analog voltage to digital code (or ADC) transfer function is not perfect since it relies on real electronic components. An assessment of the linearity of all 67 FADC cards was made and is reported in Section 4.1. This gives a general limit on the achievable linearity performance for a single channel.

We note the presence of a low amplitude high frequency noise shown in Section 4.2, which could also affect the charge measurement.

The choice of dynamic range, signal amplitude and noise level, has an impact on the overall charge linearity achievable. This is an important issue for the 8-bit FADC. This is discussed in Section 5 and illustrated with tests with a single channel of the Double Chooz electronics, including PMT pulsed with an LED. Measurements of the gain with different light levels, gains and noise levels, were made using the well known photostatistics technique described in 12 .

\subsection{FADC Linearity}

There are two values important in the definition of the linearity of an ADC; the first, is the Differential Non-Linearity (DNL), and the second is the Integral Non-Linearity (INL). These parameters determine the voltage-to-ADC transfer function, how an analog voltage input is converted to a digital code (or ADC value).

The DNL is the measure of the deviation from the ideal step size of 1 ADC count (or Least Significant Bit). The DNL for each ADC count can be positive (so the step size is larger than ideal) or negative (so the step is smaller than ideal). The INL is the cumulative effect of the DNL. It is the difference between the FADC and an ideal voltage-to-ADC transfer function. For a good discussion of FADC linearity see for example [13, 14]. 

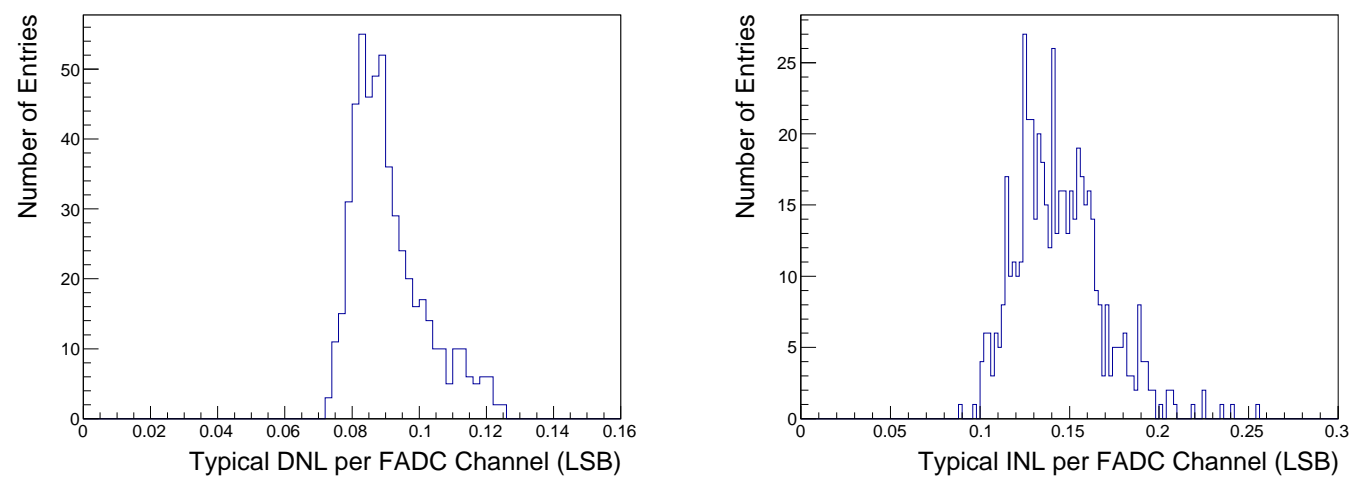

Figure 2. Non-Linearity measurements of all channels. On the left, the typical DNL per channel and on the right, the typical INL per channel.

Measurements of the DNL for each code of 536 FADC channels were made using a simple histogram method. A $1 \mathrm{~V} 12$ bit DAC, provided by one FADC card, was used as a controllable source of DC voltage giving 4096 incremental steps of $244 \mu \mathrm{V}$, into the input of each FADC channel. For each voltage input, a waveform of 1024 samples was recorded. The input voltages span a range between $2 \mathrm{mV}$ and $1.02 \mathrm{~V}$, allowing measurements of a large fraction of each FADC channel range (from ADC code 20 to 245$)$. The short step voltage $(244 \mu \mathrm{V})$ resulted in the repeated sampling of all ADC codes in the measured range. The 4096 waveforms recorded were used to make a histogram of the sampled ADC codes. A perfect FADC would show a uniform sampling of all ADC codes, distortions to the histogram indicates a variation in the code width. In this way the DNL per code was estimated. As the INL is the cumulation of each DNL per code, the INL per code was calculated from this data.

Each FADC channel was assessed by calculating the standard deviation of the obtained DNL and INL values, as shown in Figure 2. The average of each histogram is used to estimate the typical value of the FADC chip. The maximum DNL and INL values for each channel were also found to assess the extremes. In our sample of cards, we find that the typical DNL value is $\sim 0.09 \mathrm{LSB}$, with a maximum of $0.45 \mathrm{LSB}$. Similarly for the INL, the typical value is $\sim 0.26 \mathrm{LSB}$ with a maximum of $0.7 \mathrm{LSB}$. All values obtained are well within the specifications of the manufacturer, where the typical maximum DNL value is $0.16 \mathrm{LSB}$ with limits of $\pm 0.6 \mathrm{LSB}$ and typical $I N L$ of $0.3 \mathrm{LSB}$ with limits at \pm 0.9 LSB[15].

\subsection{High Frequency Correlated Noise}

The input noise to the FADC for the Double Chooz electronics is low, with a measured RMS of $\sim 1.2 \mathrm{mV}$. Upon averaging of successively triggered waveforms, low amplitude repeating signals become visible, as can be seen in Figure 3. These signals are only observable when the waveforms are averaged (their amplitudes are far below 1 LSB), so they occur synchronously to the FADC clocks. The $16 \mathrm{~ns}$ duration of these signals could correspond to the oscillation of the main $62.5 \mathrm{MHz}$ clock. The faster oscillations observed, could be harmonics from the $500 \mathrm{MHz}$ FADC sampling clock. Correlated noise on the baseline is not uncommon for boards containing high speed clocks, 


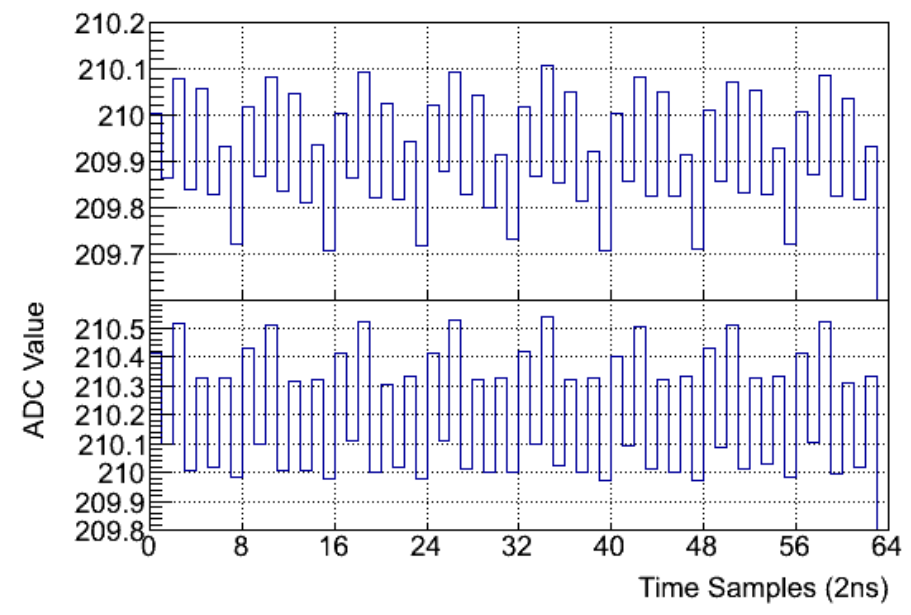

Figure 3. Averaged waveforms showing repeated patterns which correspond to the frequencies and harmonics of the FADC clocks.

and has been observed in many experiments. The magnitude of these signals vary from channel to channel and from card to card.

\section{Digitisation}

In the digitisation of an analog waveform, two discretisations occur:

1. sampling: discretisation of the time

2. quantisation: discretisation of the amplitude

If certain conditions are met on both the sampling and quantisation, then the analog signal can be perfectly reconstructed from the digitised waveform. In this case of no-distortion, measurements of parameters such as the integrated pulse charge on the digital waveform yield consistent results as if they had been made with the original analog signal. The condition for sampling was described by Shannon in 1948 and is known as the Sampling Theorem. Far less well publicised is the condition on the quantisation which was developed by Widrow in the late 1950s [16].

For the application of Double Chooz, the use of FADCs to record scintillator pulse shapes from PMTs, the speed of the PMTs and scintillator coupled with the bandwidth limitation of the FrontEnd Amplifier and FADC ( $<200 \mathrm{MHz}$ ) ensures that the condition of the Sampling Theorem is met (sampling at $500 \mathrm{MHz}$ ). The Quantisation condition, however, requires attention to the amplitude (and form) of the signal and the analog noise level.

During operation of the Double Chooz far detector, it was observed that each time the DC offset of the FADCs was re-adjusted, not only the pedestal values changed slightly (which was expected), but also the determined gain of each channel appeared to change. Also an unexpected non-linear energy response was found for each individual channel. We performed extensive studies, both experimental and by simulation, of the effects in play, and found that the dominant effect was not ADC non-linearity, but quantisation-induced non-linearity. 
The charge contained in a signal is calculated by summing the difference between consecutive current samples and the determined baseline. The photoelectron signal is extremely variable and the noise induced by the quantisation of the pulse shape is relatively small. The baseline, however, is observed to be extremely stable such that any bias on its knowledge results in a systematic bias on the charge. The correct determination of the baseline is linked to the analog noise level. When the noise is large, in comparison to the ADC step (or $1 \mathrm{LSB}$ ), the baseline can be well determined. Conversely, when the noise level is low, the baseline position is not well known. Good quantisation of the baseline, we find, is extremely important. The following describes this effect in more detail.

\subsection{Quantisation}

Widrow derived two Quantisation Theorems (QT1 and QT2) linking the signal Probability Density Function and the quantisation step size $q$ which for a Flash ADC would be the voltage (or current) difference between two successive digital values: the ADC step (1 LSB). The first theorem, QT1, describes the conditions for which there is an unique relation between the statistical descriptions of the input and output signals of the quantiser. The second, QT2, is a looser condition which, if met, ensures that at least the moments of the quantised variable are equal to the moments of the sum of the input variable and a uniformly distributed noise. For a good discussion on this subject see [17].

When measuring the signal baseline with a Flash ADC, the signal is the DC offset plus the analog noise, which, in this case, is observed to be Gaussian noise, with a standard deviation $\sigma_{\text {noise }}$. If $\sigma_{\text {noise }}$ is larger than the quantisation step $q$ then QT1 is fulfilled, and complete reconstruction of the waveform baseline can be made from the digitised version. If $\sigma_{\text {noise }}>\frac{q}{2}$ then QT2 is fulfilled, and the estimated mean and variance of the pedestal are equivalent to the input mean and variance.

If QT2 is not fulfilled, the estimated mean and variance of each waveform baseline will be biassed. In this case, the real DC offset of the baseline will not be equal to the true offset, and the measured noise level will also be wrong.

The derivations for these biases for several distributions including the Gaussian case can be found in [18]. Here, are reproduced the equations related to the digital and analog mean and variance of a Gaussian distribution. The bias observed is related to the analog RMS noise level $\left(\sigma_{n}\right)$ expressed as a fraction of the quantisation step $q$, and the true offset of the waveform $e_{q}$. The observed digitised mean offset $\left(m_{e}\left(e_{q}\right)\right)$ is:

$$
m_{e}\left(e_{q}\right)=\frac{1}{\pi} e^{-2 \pi^{2} \sigma_{n}^{2}} \sin \left(2 \pi e_{q}\right), \sigma_{n} \geq 0.3
$$

and the digitised output variance is:

$$
\sigma^{2}=\frac{1}{12}+\sigma_{n}^{2}-e^{-2 \pi^{2} \sigma_{n}^{2}}\left(\left(4 \sigma_{n}^{2}+\frac{1}{\pi^{2}}\right) \cos \left(2 \pi e_{q}\right)-\frac{1}{\pi^{2}} e^{-2 \pi^{2} \sigma_{n}^{2}} \sin ^{2}\left(2 \pi e_{q}\right)\right), \sigma_{n} \geq 0.3
$$

From equation 5.1, the bias on the measured baseline varies with the true DC offset of the waveform $e_{q}$. The distortion on the waveform is different for a signal (such as a photoelectron or scintillation pulse) than for the baseline. Observable signals use more ADC codes to describe them, and are inherently variable. So that we can consider that the main cause of bias on the measurement of pulse charge occurs due to the waveform baseline (or pedestal). This bias is simply proportional to the number of samples used to integrate the signal and the relative value of this bias depends on 

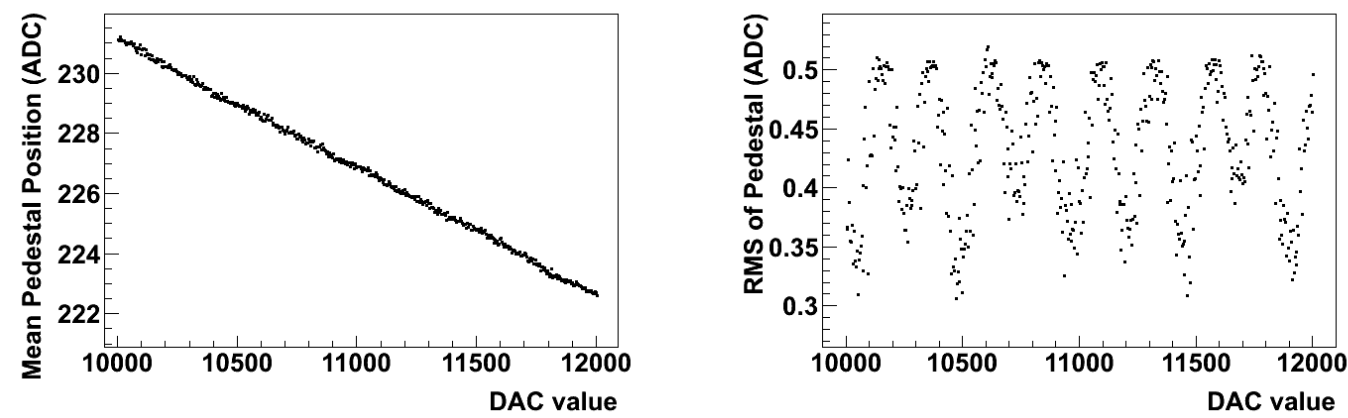

Figure 4. Example of a DAC scan of a Flash ADC channel crossing 8 consecutive ADC codes. On the left, the estimated pedestal mean is shown as a function of the input DC offset (DAC) which is proportional to DC offset voltage and on the right, the waveform RMS.

the signal amplitude. If the signal amplitude is high, this bias can be small. Conversely if the signal amplitude is low, then this bias can be significant.

A mismatch between the analog signal, in gain and noise level, and the quantisation step of a waveform digitiser can lead to a significant non-linearity on the measurement of pulse charge. A tell-tale sign of this problem is the effect described in 5: a shift in the DC offset, which is most often caused by power cycling of the electronics, results in an apparent shift in the measured single photoelectron gain. The first phase of running of the Double Chooz far detector, suffers from this digitisation problem which is now experimentaly validated with measurements from a single channel test setup.

\subsection{Experimental Study of Quantisation Effects}

Firstly the DC offset is incremented using the 16-bit controllable DAC whose value is proportional to DC voltage. For each DAC value the mean and RMS of the acquired waveforms are plotted in Figure 4 . Oscillatory patterns are observed for the estimated pedestal mean, as expected from Equation 5.1, and the measured RMS, which oscillates according to Equation 5.2.

To test the charge performance, we considered two scenarios; the first is the standard Double Chooz electronics and gain, with an RMS noise level of $1.2 \mathrm{mV}$ and a typical mean single photoelectron amplitude of $35 \mathrm{mV}$, the second is with a factor of two higher gain and with a RMS noise level of $3 \mathrm{mV}$. A FADC card is triggered by the pulse generator each time the LED illuminates. The mean number of photons per shot is tuned so that all pulses are well-contained within the 8-bit range, and no signals are saturated. For each LED setting, the DC offset (controlled by the 16-bit DAC) is shifted and 25,000 events are recorded. The DC offset is moved such that the waveform baseline crosses 3 complete ADC codes. Data was taken for different levels of LED illumination. Figure 5 shows two example waveforms of low light-level signals taken under the two conditions of gain and noise.

The RMS noise levels were measured, and are shown in Figure 6. At high gain and high noise, a small variation of the baseline noise level is observed with DC offset, which is most likely due to the variation of code size (DNL). For the low gain and low noise scenario, a large cyclic varation, corresponding to the transition of the ADC codes, of the RMS noise level is observed. 

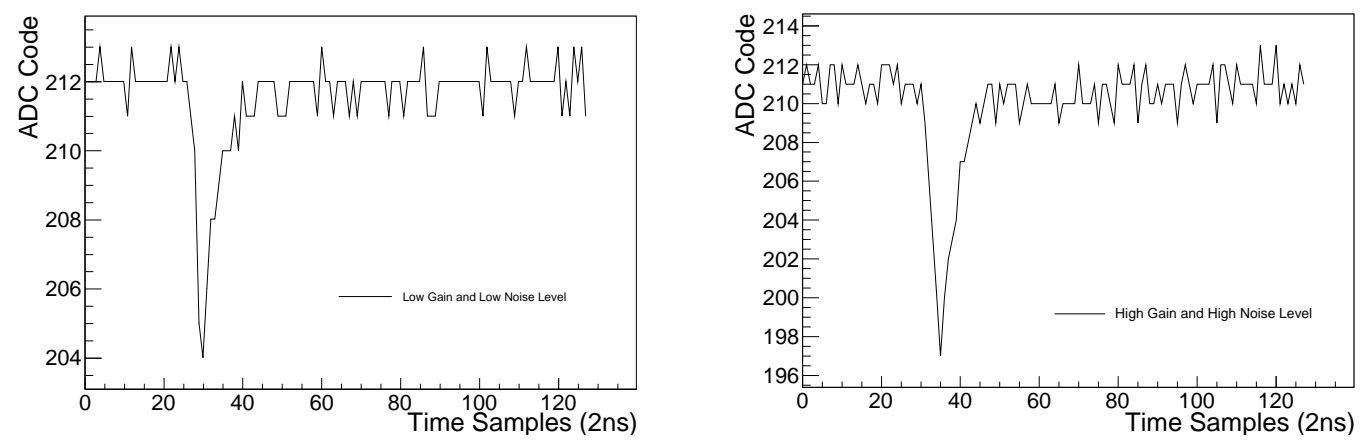

Figure 5. Examples of waveforms for the case of low gain and low noise (left) and for higher gain and higher noise conditions (right).
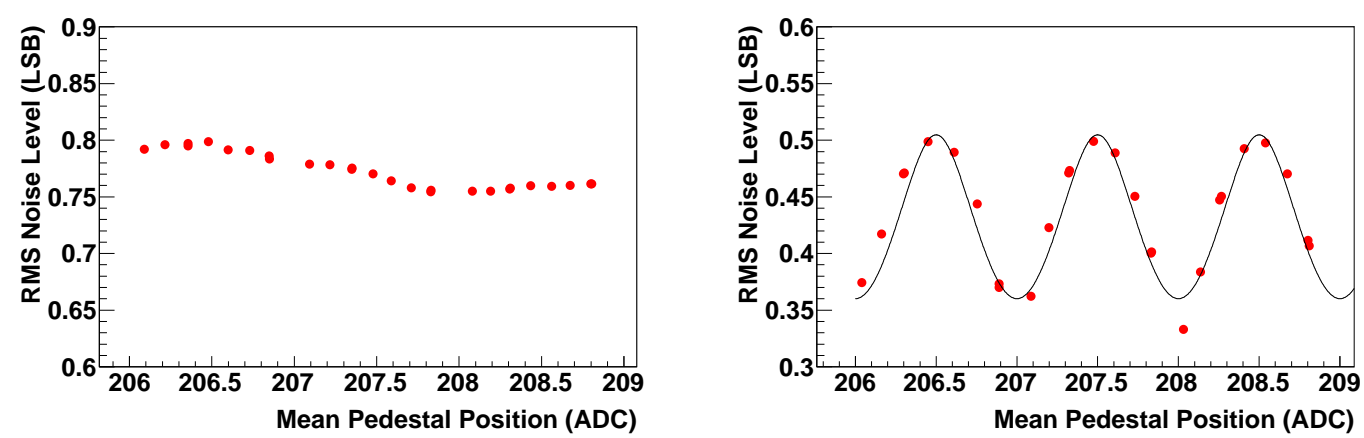

Figure 6. RMS Noise levels at different DC offsets. Left, the higher analog noise level used for the high gain runs. Right, the lower noise case, where a clear oscillatory trend is observed overlaid with the expected trend assuming a real RMS noise level of 0.33 LSB.

Figure 7 shows the measured gains for these two conditions. Under good running conditions, the measured gain is expected to be consistent for all values of the DAC offset. The Kuiper test was used, as it is sensitive to cyclic variations, to search for deviations from the expectation that the gain is constant with a significance level of 0.05 . For the high gain and high noise level case, the measured gains are consistent for all values of the DC offset and vary little with light level. In the low gain and low noise case, however, large cyclic variations, corresponding to the transitions of ADC code, are observed in the measured gain, at low light levels, becoming less prominent with increasing light levels.

For the low gain and low noise case, as the signals become larger, the bias on the baseline becomes less significant in comparison to the contained charge, and the oscillations dampen. The shape of the oscillations, the position of minima and maxima, is complicated by the variation of the code size (DNL) and also by the presence of correlated high frequency noise. It is clear that a strong charge non-linearity is present whose magnitude (and sign) is dependent on the pedestal position (DC offset). In this case, the gain measured at the single photoelectron level can be significantly different to that measured at higher light levels. 

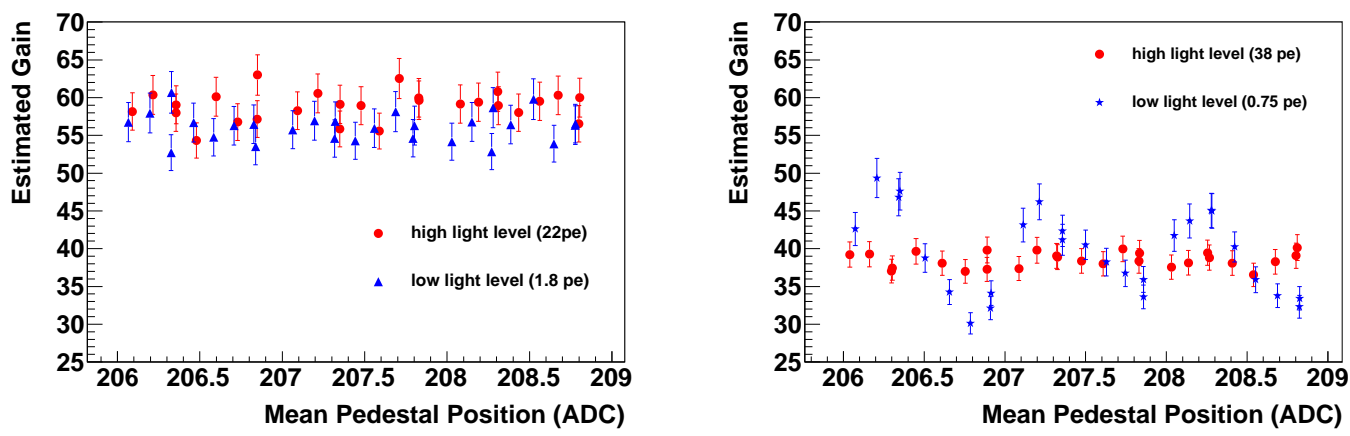

Figure 7. Gain measurements at different DC offsets and light levels. Left, the high gain and higher analog noise level, resulting in consistent gain measurements for all light levels (filled circles and triangles with means of $\sim 22$ and 1.8 photoelectrons per LED shot respectively). Right, the lower gain and noise case, where a clear oscillatory trend is observed with a greater amplitude for lower light levels (filled circles and stars with means of $\sim 38$ and 0.75 photoelectrons respectively).
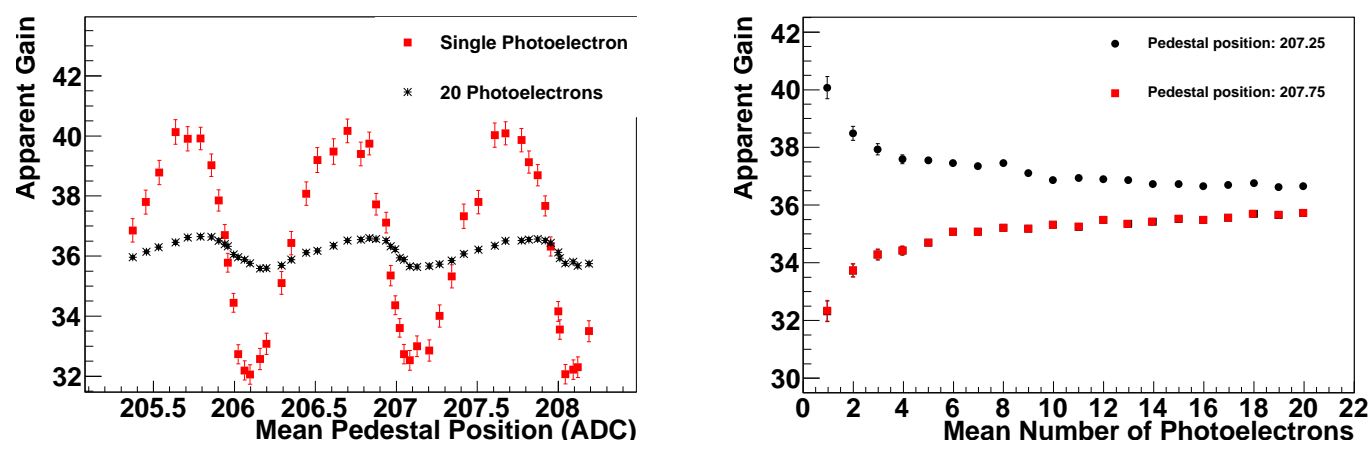

Figure 8. Example from simulation. Left, variation of apparent gain as a function of pedestal position for 1 photoelectron (filled squares) and 20 photoelectrons (points). Right, the variation of apparent gain with increasing number of photoelectrons. Here, shown for two values of pedestal position, 207.25 (filled circles) and 207.75 (filled squares). 


\section{Simulation}

The described effects can be well reproduced by simulation. Waveforms are generated, first in an analog sense (in volts) and then 'digitised' using a voltage-to-ADC transfer function which represents a typical FADC. The FADC behaviour can be chosen to be perfectly linear or imperfect ie using measured DNL values or with randomly chosen code widths (whilst obeying the typical values of maximum INL and DNL of the FADC chip as measured in Section 4.1). The analog part of the simulation was tuned to represent the behaviour of the PMT and Front-End Amplifier, with typical RMS noise levels. Also included was the high frequency clock noise, using the observed patterns shown in Section 4.2. Single photoelectrons were generated such that the mean gain as observed by the FADC is $\sim 36$ LSB $x 2 \mathrm{~ns}$. The single photoelectron charge distribution was assumed to be Gaussian with sigma of $35 \%$. The temporal form of the single photoelectron is given by a Landau distibution, with parameters tuned to match the actual time profile of a single photoelectron. The LED pulse profile was generated assuming a square pulse.

Figure 8 shows an example of the simulation results. Here, a perfectly linear FADC was simulated with an analog noise level of $1 \mathrm{mV}$ and the high frequency noise amplitude of $0.4 \mathrm{mV}$ (approximately 0.1 LSB). A clear oscillation in gain is found for low light levels which diminishes in strength for higher light levels. Fixing the waveform pedestal position and plotting the measured gain as a function of increasing number of photoelectrons shows clearly the charge non-linearity, shown here for two extreme pedestal positions. Depending on the pedestal position, the measured gain can either increase or decrease with increasing light level. Increasing the RMS noise level to $2 \mathrm{mV}$, as predicted by QT 2, reduces the charge biases to insignificant levels.

\section{Conclusion}

The waveform digitiser of the Double Chooz experiment was presented. In all 66 cards divided into $5 \mathrm{VME}$ crates are required to form the data acquisition system of the experiment. A dedicated firmware allows the synchronous running of these cards, operating with no deadtime at trigger rates of $\sim 150$ events/s. This firmware also has a flexible readout capability, allowing on-the-fly decision making on the read-out duration of the waveforms. These functions are ideal for rare event searches, such as neutrino reactor experiments, where the rate of the signals of interest are dominated by the rate of background events.

The general linearity of the card was assessed through measurements of the Differential NonLinearity and Integral Non-Linearity of 67 eight-channel FADC cards. These measurements aided in a more specific study of the systematic biases on the determination of the pulse charge from signals from a PMT tube.

The use of an 8-bit FADC operated in a high dynamic range was described, where signals vary per channel from 1 to $\sim 50$ photoelectrons. Sources of systematic bias were shown for the case where the signal amplification and analog noise level is low. In this regime, biases from clockcorrelated noise, quantisation and intrinsic non-linearity of the FADC chip (DNL and INL) are important. These effects were found to be well reproducable by simulation. Problems, that can occur if the analog signal and noise level are not correctly matched to the digitisation step, were explored. We show, the gain and noise level required to adequately eliminate these problems. 
It is interesting to note that the Sampling Condition (from the Sampling Theorem) is generally taken into account into the design of a waveform digitiser, but the Quantisation condition is not. It is an error to consider that noise is always a bad thing. Noise is a dithering agent, and, as such, is necessary. We recommend that manufacturers provide a means to enable/disable, or, even better, adjust a source of Gaussian noise to add to the analog input signal, so as to give the possibility to reach a baseline RMS equal to 0.5 LSB.

\section{References}

[1] Y. Abe et al, Indication for the disappearance of reactor electron antineutrinos in the Double Chooz experiment, PRL 108 (2012) 131801.

[2] Y. Abe et al, Reactor electron antineutrino disappearance in the Double Chooz experiment, PRD 86, (2012) 052008.

[3] C. Aberle et al, Large scale Gd-beta-diketonate based organic liquid scintillator production for antineutrino detection, 2012 JINST 7 P06008.

[4] Hamamatsu Corporation, Large Photocathode Area Photomulitplier Tubes.]

[5] C. Bauer et al, Qualification Tests of 474 Photomultiplier Tubes for the Inner Detector of the Double Chooz Experiment, 2011 JINST 6 P06008.

[6] T. Matsubara et al, Evaluation of 400 low background 10-in. photo-multiplier tubes for the Double Chooz experiment, NIM A 661 (2012)16-25.

[7] K. Zbiri, Note on Drexel tests of the IMB R1408 PMTs used in the inner veto of both far and near detectors of the Double Chooz experiment arXiv:1104.4045 (2011).

[8] T. Akiri, PhD Thesis, (2010).

[9] Emerson Network Power, MVME3100 Data Sheet].

[10] CAEN, V1721 Data Sheet [.]

[11] R. Acciarri et al, Tests of PMT Signal Read-out in a Liquid Argon Dark Matter Detector with a New Fast Waveform Digitizer, 2012 JINST 7 P07003.

[12] F.J. Lombard and F. Martin, Statistics of Electron Multiplication, Rev. Sci. Instrum. 32 (1961) 200.

[13] W. Kester, Testing Data Converters, Section 5.2: Testing ADCs.]

[14] Tutorial 283, INL/DNL Measurements for High-Speed Analog-to-Digital Converters (ADCs).

[15] National Semiconductor,ADC08D500 Data Sheet.

[16] B. Widrow et al, IEEE Trans. Instrumentation and Measurement, 45, (1996) 353-361.

[17] B. Widrow and I. Kollar, Quantization Noise: Roundoff Error in Digital Computation, Signal Processing, Control, and Communications, Cambridge University Press 2008.

[18] P. Carbone and D. Petri, Mean value and variance of noisy quantized data, Measurement, 23 (1998) 131-144. 